\title{
The Role of Personality and Job Stressors in Predicting Counterproductive Work Behavior: A three-way interaction
}

\section{Zhiqing E. Zhou*, Laurenz L. Meier** and Paul E. Spector*}

*Department of Psychology, PCD 4118, University of South Florida, Tampa, FL 33620, USA.

zhiqing@mail.usf.edu

**University of Fribourg, Fribourg, Switzerland

The current study examined interactive effects among personality and job stressors in predicting employees' engagement in counterproductive work behavior (CWB) defined as behavior that harms organizations or people in organizations. Survey data were collected from 932 employees and results showed significant negative relationships of agreeableness, conscientiousness, and emotional stability with CWB directed at organizations (CWB-O) and people (CWB-P), and significant positive relationships of interpersonal conflict and organizational constraints with CWB-O and CWB-P. Further, it was found that the positive relationships of interpersonal conflict with CWB-O and CWB-P were strongest for people of low emotional stability-low agreeableness among all emotional stability-agreeableness combinations, and that the positive relationships of organizational constraints with CWB-O and CWB-P were strongest for people of high emotional stability-low conscientiousness among all emotional stability-conscientiousness combinations.

\section{Introduction}

ounterproductive work behavior (CWB) refers to intentional behaviors that harm or intend to harm organizations or people in organizations (Spector \& Fox, 2005). Examples of CWB include yelling at someone, stealing from the organizations, damaging organization's property, and taking longer breaks than allowed. Considering the nature of these behaviors, it is of little doubt that CWB can have important negative impact on both organizations and people in organizations (Bowling \& Eschleman, 2010; Camara \& Schneider, 1994; Rotundo \& Spector, 2010; Vardi \& Weitz, 2004). Because of concerns over potential the negative effects, past research has taken different approaches to uncover possible predictors of CWB that might enhance our understanding on why employees engage in CWB. Traditionally, while some studies have focused on main effects of job stressors and personality (e.g., Hershcovis et al., 2007; Salgado, 2002), others have examined two-way interactive effects between job stressors and personality in predicting CWB (e.g., Bowling \& Eschleman, 2010; Fox, Spector, \& Miles, 2001; Penney, 2002; Penney \& Spector,
2005). Building on these findings, a personalitymoderated stressor-CWB relationship has been well established. More recently, a few researchers have started to pay attention to prediction of CWB with combinations of several personality traits (Bowling, Burns, Stewart, \& Gruys, 2011; Jensen \& Patel, 2011), but what is lacking in the current literature is an integration of the combined personality approach with the stressor-personality interaction approach in predicting CWB. That is, in addition to individual personality traits, whether certain combinations of personality traits also influence previously known stressor-CWB relationships, is a question we want to address in the current study. Thus our study contributes to the current literature of CWB by further exploring patterns in which individuals react to experienced job stressors with CWB.

\section{Main effects and two-way interaction of stressors and personality on CWB}

CWB consists of behaviors that have been divided into two categories by the target (Robinson \& Bennett, 1995). CWB against organizations (CWB-O) consists of 
acts from minor behaviors, such as taking longer breaks than allowed, to more severe acts such as stealing organizational property. CWB against people (CWB-P) includes mainly aggressive acts which can be either physical (e.g., hitting someone) or nonphysical (e.g., insulting someone). This taxonomy has been widely used in CWB research and thus will be applied in the current study.

A great amount of research has been carried out to uncover external and internal antecedents of CWB. Externally, it has been suggested that stressful events and conditions at work (job stressors) can cause employees to react with negative emotions and behaviors (Spector \& Jex, 1998), and that perception of workplace stressors can lead to engaging in CWB (Cullen \& Sackett, 2003). Past research has provided consistent evidence for direct positive relationships of CWB with a variety of job stressors, specifically distributive injustice, procedural injustice, interpersonal conflict, and organizational constraints (Hershcovis et al., 2007). In a meta-analysis, organizational constraints and interpersonal conflict were found to have the largest mean correlations with CWB (Hershcovis et al., 2007), and thus they were our choices of job stressors in the current study. Organizational constraints refer to situations or events at work that interfere with one's efforts to complete his/her work. Examples include poor equipment and inadequate training. Interpersonal conflict concerns the frequency of arguments or disputes one has with others at work (Spector \& Jex, 1998).

Another line of research focuses on internal predictors of CWB and it has been well established that people of certain personality traits are more likely to engage in CWB. Among various personality models, the five-factor model has been widely used in CWB literature (e.g., Berry, Ones, \& Sackett, 2007; Sackett \& DeVore, 2002). Among the five factors, agreeableness, conscientiousness, and emotional stability have been studied the most (e.g., Bowling et al., 2011; Bowling \& Eschleman, 2010; Jensen \& Patel, 2011). Meta-analyses provided evidence that agreeableness, conscientiousness, and emotional stability all negatively predicted CWB, whereas extraversion and openness failed to relate to CWB (Berry et al., 2007; Salgado, 2002). People high in agreeableness are more cooperative, people of high conscientiousness are more responsible and cautious, and people of high emotional stability are less likely to experience negative emotions. It was suggested that people with these traits are more likely to believe CWB can be detected, less likely to believe that other employees are engaging CWB, and are more motivated to comply with workplace norms, and thus engage in less CWB (Jensen \& Patel, 2011). Given the fact that agreeableness, conscientiousness, and emotional stability were suggested to have the most potential to predict CWB (Jensen \& Patel, 2011) and extraversion and open- ness to experience were not related to CWB (Berry et al., 2007), the current study will only include agreeableness, conscientiousness, and emotional stability as internal personality predictors of CWB.

A third approach has examined joint effects of stressors and personality traits in predicting CWB. For example, Fox et al. (2001) found that trait anxiety (a personality trait closely related to emotional stability) moderated the positive relationship between interpersonal conflict and CWB-P while trait anger moderated the positive relationship between organizational constraints and CWB-O, with the positive relationships being stronger for people at high level as compared with low level of these traits. Further, Penney and Spector (2005) found that negative affectivity (another trait related closely to emotional stability) moderated the relationships of interpersonal conflict and organizational constraints with CWB, with the positive relationships being stronger for people of high as compared with low negative affectivity. Recently, Bowling and Eschleman (2010) reported that the positive relationships of interpersonal conflict and organizational constraints with CWB were stronger when employees were low on agreeableness, conscientiousness, and emotional stability, respectively.

Bowling and Eschleman (2010) discussed the potential reasons for the moderating effect of agreeableness, conscientiousness, and emotional stability on stressorCWB relationships. The first possible explanation focuses on strategies people of different levels of personality traits might take when facing job stressors: engaging in CWB might be the immediate response strategy for people at low levels of these personality traits when facing job stressors, while people of high levels of these personality traits tend to engage in other more effective coping responses and will engage in CWB only after the constructive coping responses do not work. For example, people of low conscientiousness might take engaging in CWB as their immediate response after experiencing job stressors, while people of high conscientiousness would first try to figure out whether they can deal with the stressors in more effective ways, and might engage in CWB only after all possible effective ways have failed. Their second possible explanation concerns threshold for CWB, and proposes that people at low levels of these personality traits have low thresholds for CWB and tend to engage in CWB even when experiencing low levels of job stressors, while people at high levels of these personality traits have higher thresholds for CWB and will engage in CWB only when facing high levels of job stressors. For example, people of low agreeableness might have low threshold for CWB and would engage in CWB when experiencing low levels of job stressors; on the other hand, people of high agreeableness might have high threshold for CWB and would engage in CWB only 
when facing high levels of job stressors. Taken together, past findings have provided empirical evidence and potential theoretical underlying mechanisms for the moderating effects of individual personality traits on stressor-CWB relationships.

\section{Combined personality on CWB and stressor-CWB relationship}

Previous research has mainly focused on effects of individual personality traits, either main effects or moderating effects, in predicting CWB, which assumes that the effect of one personality trait is not affected by levels of a second trait. In contrast, Epstein (1994) suggested that it is necessary to examine how personality variables interact with each other within individuals to better understand people's behavior. This approach can help us better understand the stressor-CWB relationships given the fact that people of different combinations of personality variables tend to engage in different coping strategies, and to experience different levels of negative attitudes (Grant \& Langan-Fox, 2006; Vollrath \& Torgerse, 2000). For example, Grant and Langan-Fox (2006) found that people of low emotional stability and low conscientiousness tend to engage in more dysfunctional coping (e.g., behavioral disengagement) instead of problem-focused coping, and to experience more job dissatisfaction. Meanwhile, people of low emotional stability and low agreeableness also tend to have higher job dissatisfaction. It is possible that individuals of different combinations of personality traits might have different coping strategies and different tendencies to experienced negative attitudes when experiencing job stressors.

Along with this idea, more recently, a few researchers have explored two-way interactions among personality traits in predicting CWB. For example, Jensen and Patel (2011) found that all three two-way interactions among agreeableness, conscientiousness, and emotional stability predicted CWB above and beyond their individual main effects. Specifically, when employees were high on both traits in any combination, they engaged in the least CWB. Bowling et al. (2011) also reported that the negative relationship between emotional stability and CWB was stronger for participants who were low on either conscientiousness or agreeableness than for those who were high on those traits. These findings suggest that the tendency to engage in CWB not only depends on one single personality trait, but also on the interactions between them.

It is reasonable to believe that the interaction among these personality traits might shed some light on the stressor-CWB relationships. When employees experience job stressors at work, CWB can be one way of coping (Cullen \& Sackett, 2003). That is, when employees experience job stressors at work, if they cannot en- gage in effective coping strategies, such as problemfocused coping to change the stressful situations, they are more likely to engage in alternative coping strategies such as performing CWB. Grant and Langan-Fox (2006) and Vollrath and Torgerse (2000) have both found that people of low emotional stability-low conscientiousness use more dysfunctional coping and less problem-focused coping, so it is likely that people with this personality trait combination will also engage in the most CWB when experiencing job stressors. One plausible explanation is that although people of low emotional stability are more likely to engage in avoidance-based coping instead of problem-focused coping (Cullen \& Sackett, 2003), this tendency is also subject to regulations of other personality traits such as conscientiousness. Compared with people of low conscientiousness, people of high conscientiousness tend to be more self-disciplined and dutiful, which encourages them to engage in more problem-focused coping and to think more about the potential consequences of engaging in CWB, and thus restricts their tendency to engage in CWB. Therefore, for a person of low emotional stability but of high conscientiousness, the restricting effect of high conscientiousness will inhibit his or her tendency to engage in more dysfunctional coping and subsequent harmful behaviors; on the other hand, a person of low emotional stability and low conscientiousness will be less restricted and is more likely to engage in dysfunctional coping such as more CWB. Further, as people of high agreeableness are low on the tendency to harm others (Bowling et al., 2011) and high on the tendency to get along with others, it is likely that high agreeableness will have the same restricting effect on low emotional stability by avoiding the potential negative consequences of engaging in CWB and encouraging them to use more effective coping.

Moreover, the stressor-CWB relationships are also subject to people's tendency to experience negative emotions with the relationships being stronger for people who are more likely to experience negative emotions (Penney \& Spector, 2005). Grant and Langan-Fox (2006) found that people of low emotional stability-low conscientiousness and people of low emotional stability-low agreeableness are both more likely to experience job dissatisfaction. As people of low emotional stability have the tendency to experience negative emotions in general, it is likely that low conscientiousness or low agreeableness will strengthen this tendency when experiencing job stressors, while high conscientiousness or agreeableness will motivate people to focus more on dealing with their problems, and thus experience less negative emotions. Therefore, it is reasonable to speculate that people of low emotional stability-low conscientiousness and people of low emotional stabilitylow agreeableness are more likely to experience negative emotions when experiencing job stressors, and then subsequently engage in more CWB. 
In summary, when experiencing job stressors, people of low emotional stability will react with more CWB in general (Bowling and Eschleman, 2010), but it is likely that this reaction will be stronger when they are also low on conscientiousness or low on agreeableness as compared with when they are high on conscientiousness or high on agreeableness, respectively. Taken together, we expect that the stressor-CWB relationships will be moderated by combinations of personality traits, which leads us to the following two hypotheses:

Hypothesis 1: There will be a three-way interaction among job stressors (interpersonal conflict and organizational constraints), emotional stability, and agreeableness in predicting CWB. Specifically, the positive relationship between job stressors (interpersonal conflict and organizational constraints) and CWB will be strongest for people of low emotional stability-low agreeableness among people of all emotional stabilityagreeableness combinations.

Hypothesis 2: There will be a three-way interaction among job stressors (interpersonal conflict and organizational constraints), emotional stability, and conscientiousness in predicting CWB. Specifically, the positive relationship between job stressors (interpersonal conflict and organizational constraints) and CWB will be strongest for people of low emotional stability-low conscientiousness among people of all emotional stabilityconscientiousness combinations.

\section{Method}

\subsection{Participants}

Data were collected from 932 employed individuals recruited from various undergraduate and graduate classes at a large southern public university. The average age of our sample was 21.77 years $(S D=4.25$, range $=18-58)$, the mean working hours every week was $26.36 \mathrm{hr}$ with a minimum requirement of $20 \mathrm{hr}(S D=7.15$, range $=20$ 60 ), and the mean tenure at their current jobs was 25.95 months $(S D=25.92$, range $=0.5-268)$. The majority of our participants were White $(62 \%)$ and female (78\%). Our participants were working with various job titles, such as customer care representative, sales representative, waitress/waiter, bartender, assistants, and teller/cashier. An additional 115 participants were dropped because they either worked fewer than $20 \mathrm{hr}$ per week, or failed to report their working hours. One participant was deleted who reported working $98 \mathrm{hr}$ per week, which was assumed to be an error.

\subsection{Measures}

\subsubsection{Interpersonal conflict}

Interpersonal conflict was assessed using the 4-item interpersonal conflict at work scale (Spector \& Jex, 1998).
Participants were asked to rate how often each of the events had happened to them on a 5 -point scale from 1 (less than once per month or never) to 5 (several times per day). A sample item is 'How often do you get into arguments with others at work?' The scale yielded an internal reliability of .85 with our sample.

\subsubsection{Organizational constraints}

Organizational constraints were assessed with the 11item organizational constraints scale (Spector \& Jex, 1998). Participants are asked to rate how often they find it difficult or impossible to do their job because of each of 11 areas on a 5-point scale from 1 (less than once per month or never) to 5 (several times per day). An example item is 'Poor equipment or supplies'. The scale had an internal reliability of .92 in the current study.

\subsubsection{Personality traits}

Agreeableness, conscientiousness, and emotional stability were all measured with 10-item measures from the international personality item pool (Goldberg et al., 2006). Participants were asked to indicate how close each item was to characterizing them on a 5-point scale from 1 (very inaccurate) to 5 (very accurate). An example item for agreeableness was 'I feel others' emotions', an example item for conscientiousness was 'I pay attention to details', and a reversely coded example item for emotional stability was 'I worry about things'. Coefficient alphas of the scales with our sample were .89 (agreeableness), .86 (conscientiousness), and .88 (emotional stability).

\subsection{4. $C W B$}

CWB was measured with the 45-item counterproductive work behavior checklist (Spector et al., 2006). Participants were asked how often they had performed each of the behaviors on their present jobs on a 5-point scale from 1 (never) to 5 (every day). CWB-O and CWB-P were each measured using 21 and 23 items, respectively. One item does not fit either subscale and was omitted. An example item for CWB-O is 'Stolen something belonging to your employer', and one example item for CWB-P is 'Verbally abused someone at work'. The coefficient alphas of the scales in the current sample were .92 (CWB-O) and .96 (CWB-P).

\section{Results}

\subsection{Descriptive statistics and main effects}

Table 1 displays the descriptive statistics for all variables, including means, SDs, and coefficient alphas for each measure. All measures have coefficient alpha higher than .80 . 
Table 1. Zero-order correlations among study variables

\begin{tabular}{|c|c|c|c|c|c|c|c|c|c|c|c|c|c|c|}
\hline \multicolumn{2}{|c|}{ Variables } & \multirow{2}{*}{$\begin{array}{r}\text { Mean } \\
0.22\end{array}$} & \multirow{2}{*}{$\frac{S D}{0.41}$} & \multirow{2}{*}{$\frac{1}{-}$} & \multirow[t]{2}{*}{2} & \multirow[t]{2}{*}{3} & \multirow[t]{2}{*}{4} & \multirow[t]{2}{*}{5} & \multirow[t]{2}{*}{6} & \multirow[t]{2}{*}{7} & \multirow[t]{2}{*}{8} & \multirow[t]{2}{*}{9} & \multirow[t]{2}{*}{10} & \multirow[t]{2}{*}{11} \\
\hline 1 & Gender & & & & & & & & & & & & & \\
\hline 2 & Age & 21.77 & 4.25 & .05 & - & & & & & & & & & \\
\hline 3 & Hour & 26.36 & 7.15 & .05 & $.38 * *$ & - & & & & & & & & \\
\hline 4 & Tenure & 25.95 & 25.92 & .05 & $.36 * *$ & $.31 * *$ & - & & & & & & & \\
\hline 5 & Agreeableness & 4.06 & 0.65 & $-.20 * *$ & $.10^{* *}$ & .02 & .02 & $(.89)$ & & & & & & \\
\hline 6 & Conscientiousness & 3.67 & 0.68 & $-.11 * *$ & $.12^{* *}$ & .05 & .03 & $.33 * *$ & $(.86)$ & & & & & \\
\hline 7 & Emotional stability & 3.19 & 0.76 & $.13 * *$ & .01 & .02 & .02 & $.17^{* *}$ & $.24 * *$ & $(.88)$ & & & & \\
\hline 8 & Constraints & 1.77 & 0.75 & $.09 * *$ & .06 & $.18^{* *}$ & $.08^{*}$ & $-.16 * *$ & $-.15^{* *}$ & $-.18 * *$ & $(.92)$ & & & \\
\hline 9 & Conflict & 1.41 & 0.68 & $.10^{* *}$ & -.06 & .06 & .06 & $-.25^{* * *}$ & $-.16 * *$ & $-.16 * *$ & $.58 * *$ & $(.85)$ & & \\
\hline 10 & CWB-O & 1.54 & 0.48 & $.11 * *$ & -.02 & .04 & .06 & $-.32^{\text {*** }}$ & $-.37 * *$ & $-.21 * *$ & $.53 * *$ & .53 ** & $(.92)$ & \\
\hline 11 & CWB-P & 1.27 & 0.44 & $.17^{* *}$ & -.03 & .04 & $.07 *$ & $-.41 * *$ & $-.28 * *$ & $-.15 * *$ & $.47 * *$ & $.58^{* *}$ & $.84 * *$ & $(.96)$ \\
\hline
\end{tabular}

Note: $N=932$ for all correlations except for those involving tenure $(N=925)$. Gender: male $=1$, female $=0$; Constraints $=$ organizational constraints; Conflict $=$ interpersonal conflict; $\mathrm{CWB}-\mathrm{O}=$ counterproductive work behaviors against organizations; $\mathrm{CWB}-\mathrm{P}=$ counterproductive work behaviors against people. Cronbach's alphas are indicated in parentheses. $* p<.05, * * p<.01$.

Table 1 also shows the zero-order correlations among the study's variables, as well as demographic variables of gender, age, working hours per week, and tenure. Both job stressors were positively related to both forms CWB, indicating that people who experienced more job stressors also reported engaging in more CWB-O and CWB-P. All personality variables were negatively related to both forms of CWB, indicating that people at high level of each of the personality traits reported engaging in less CWB-O and CWB-P.

Hierarchical regression analyses were conducted to test our moderator hypotheses. Personality variables and job stressors were all centered on their grand means, and interaction terms (two-way and three-way) were computed by multiplying mean-centered predictor variables. Predictors and their interaction terms were entered in the following orders: (1) two personality traits and one job stressor; (2) two-way interaction terms of two personality variables, and two-way interaction terms of personality variables and one job stressor; (3) three-way interaction terms of two personality variables and one job stressor. Hierarchical regression analyses with and without control variables (age, gender, tenure, and working hour per week) being controlled for before step 1 were both conducted, and the patterns of significance for two-way interactions and threeway interactions remained the same. Thus, for the purpose of comparing with previous studies (e.g., Bowling \& Eschleman, 2010), results of regression analyses without control variables being controlled for are shown in Table 2. We will briefly present two-way interaction results and then concentrate on the three-way interactions.

\subsection{Two-way interactions}

As shown in Table 2, agreeableness moderated the positive relationships of interpersonal conflict and organizational constraints for both CWB-O and CWB-P, with the relationships being stronger for people of low agreeableness than people of high agreeableness. The same pattern appears for the moderating effect of conscientiousness on the positive relationships of interpersonal conflict and organizational constraints with CWB-O and CWB-P, respectively. Further, when including conscientiousness in the regression analyses, emotional stability moderated the relationships of interpersonal conflict and organizational constraints with CWB-O and CWB-P, with the positive relationships being stronger for people of high emotional stability than people of low emotional stability. Lastly, conscientiousness moderated the negative relationships of emotional stability with CWB-O and CWB-P, with the relationships being stronger for people of high conscientiousness than for people of low conscientiousness. The two-way interaction of agreeableness and emotional stability did not predict CWB-O or CWB-P in any analysis.

\subsection{Three-way interactions}

As shown in Table 2, the three-way interactions were significant for the following combinations: interpersonal conflict, emotional stability, and agreeableness interactively predicted CWB-O $(\beta=.07, p<.05)$ and CWB-P $(\beta=.06, p<.01)$; organizational constraints, emotional stability, and conscientiousness interactively predicted CWB-O $(\beta=-.07, \quad p<.01)$ and CWB-P $(\beta=-.11$, $p<.001)$. The interaction of interpersonal conflict, emotional stability, and conscientiousness and the interaction of organizational constraints, emotional stability, and agreeableness did not significantly predict CWB-O or CWB-P.

Simple slope analyses were conducted for significant three-way interactions following suggestions in Dawson and Richter (2006). Results showed that for relationships of interpersonal conflict with CWB-O and CWB-P, the slope of low emotional stability-low agreeableness combination was significantly different from 
Table 2. Regression results for three-way interaction in predicting CWB-O and CWB-P

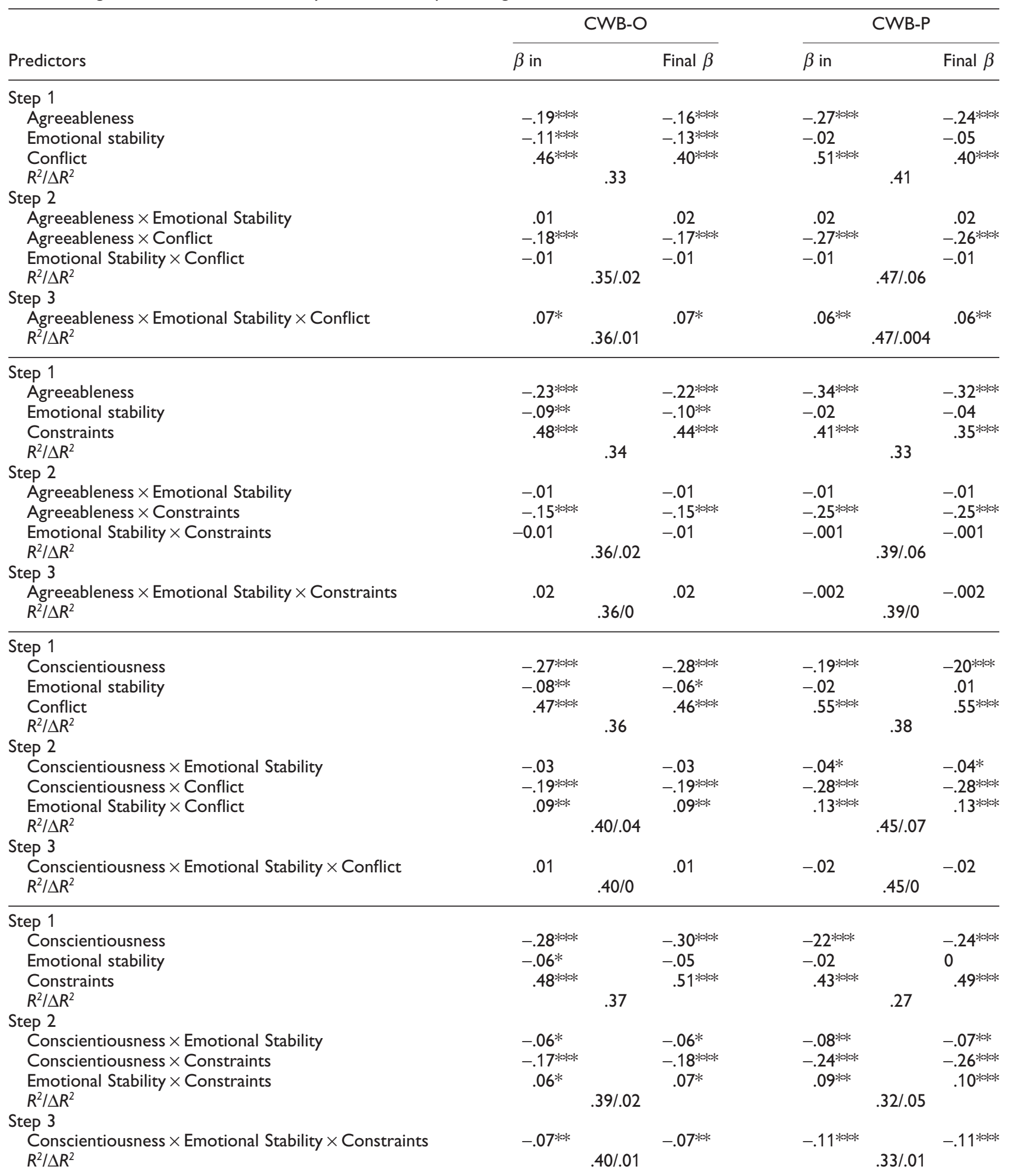

Note: $\beta$ in = Beta coefficient of the particular step at which the variable initially entered the equation from last step; Final $\beta=$ Beta coefficient when all predictors were entered; $R^{2}=$ Total amount of variance accounted for; $\Delta R^{2}=$ Amount of additional variance explained. $N=932$. Constraints $=$ organizational constraints; Conflict $=$ interpersonal conflict; $C W B-O=$ counterproductive work behaviors against organizations; CWB$\mathrm{P}=$ counterproductive work behaviors against people. $* p<.05, * * p<.01, * * * p<.001$. 


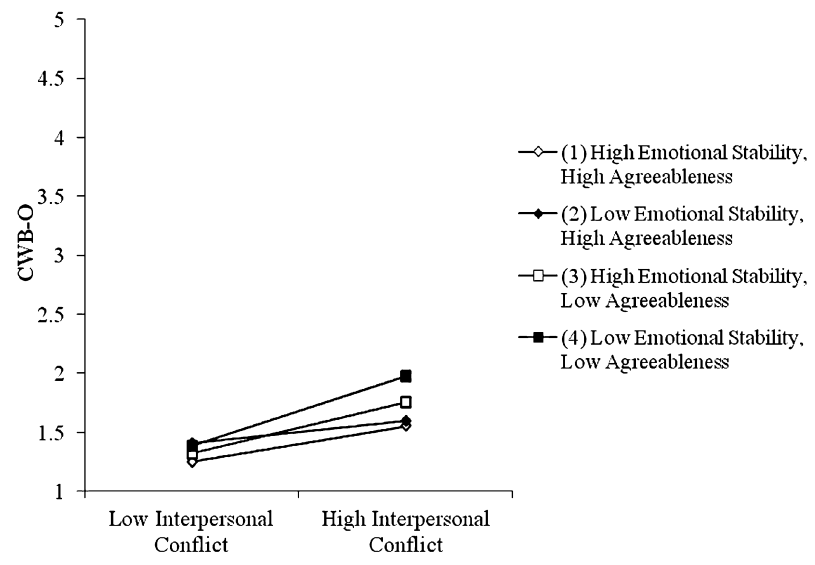

Figure 1. Interaction between interpersonal conflict, emotional stability, and agreeableness predicting counterproductive work behaviors against organizations (CWB-O).

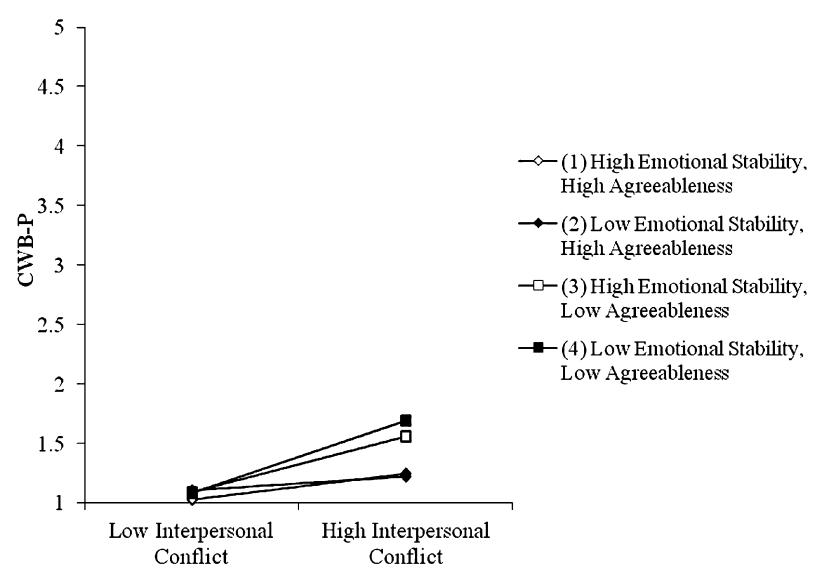

Figure 2. Interaction between interpersonal conflict, emotional stability, and agreeableness predicting counterproductive work behaviors against people (CWB-P).

slopes of other three combinations of emotional stability and agreeableness (low-high, high-low, and high-high); for relationships of organizational constraints with CWB-O and CWB-P, the slope of high emotional stability-low conscientiousness condition was significantly different from slopes of other three combinations of conscientiousness and emotional stability (low-low, low-high, and high-high).

Significant three-way interactions were plotted using unstandardized regression coefficients following procedures of Aiken and West (1991) and Dawson and Richter (2006). The patterns are shown in Figures 1 to 4. As shown in Figures 1 and 2, the positive relationships of interpersonal conflict with both CWB-O and CWB-P were strongest for people of low emotional stability and low agreeableness, thus partially supporting Hypothesis 1. Figures 3 and 4 show that the positive relationships of organizational constraints with both CWB-O and

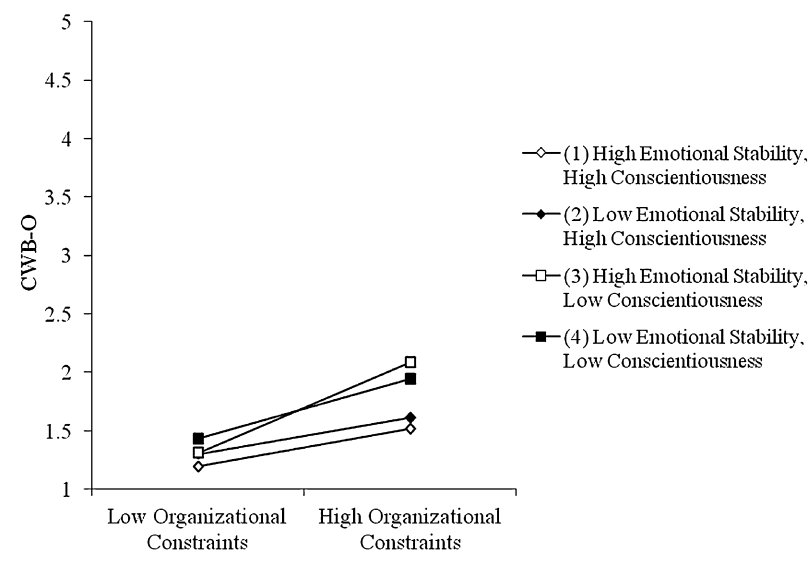

Figure 3. Interaction between organizational constraints, emotional stability, and conscientiousness predicting counterproductive work behaviors against organizations (CWB-O).

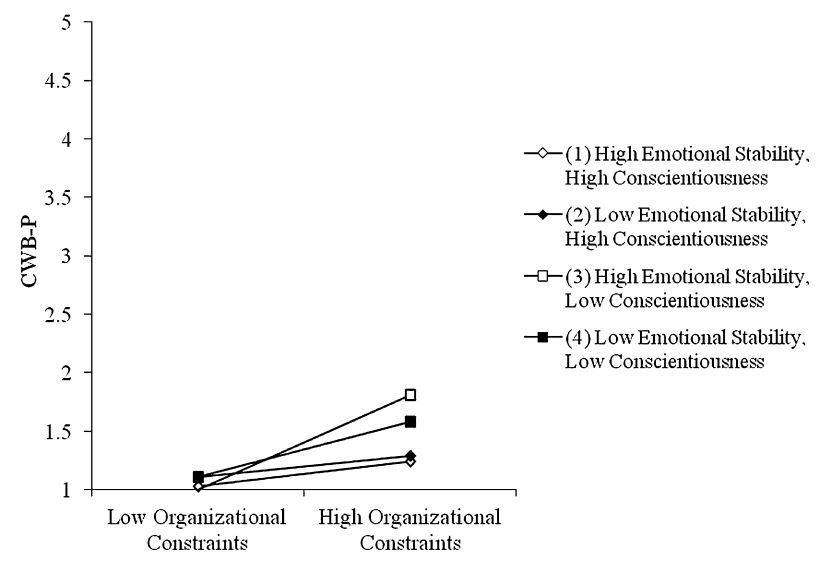

Figure 4. Interaction between organizational constraints, emotional stability, and conscientiousness predicting counterproductive work behaviors against people (CWB-P).

CWB-P were strongest for people of high emotional stability and low conscientiousness instead of low emotional stability. Hypothesis 2 was not supported.

\section{Discussion}

The current study aimed to examine three-way interactions among job stressors and personality traits in predicting CWB. We first found that both interpersonal conflict and organizational constraints were positively related with CWB-O and CWB-P, while agreeableness, conscientiousness, and emotional stability were all negatively related with CWB-O and CWB-P. Further, agreeableness, conscientiousness, and emotional stability all moderated the positive relationships of interpersonal conflict and organizational constraints with CWB-O and CWB-P, and conscientiousness moderated the relationships of emotional stability with CWB-O and CWB-P. 
More importantly, our results showed that there was a significant three-way interaction of interpersonal conflict, emotional stability, and agreeableness in predicting CWB-O and CWB-P, and also a significant three-way interaction of organizational constraints, emotional stability, and conscientiousness in predicting CWB-O and CWB-P, suggesting that the stressor-CWB relationship is moderated by certain combinations of personality traits above and beyond individual personality traits. The interaction of organizational constraints, emotional stability, and agreeableness, as well as the interaction of interpersonal conflict, emotional stability, and conscientiousness were not significant in predicting CWB-O or CWB-P.

Consistent with Berry et al. (2007), our results showed that agreeableness, conscientiousness, and emotional stability are all negatively related to CWB-O and CWB-P. Meanwhile, both interpersonal conflict and organizational constraints are positively related to CWB-O and CWB-P, which is consistent with Hershcovis et al. (2007). These results confirmed previous findings that these personality traits and job stressors are important internal and external antecedents of CWB.

The moderating effect of conscientiousness on the relationships of interpersonal conflict and organizational constraints with CWB-O and CWB-P is consistent with Bowling and Eschleman's (2010) finding, with the relationships of interpersonal conflict and CWB-O and CWB-P being stronger for people of low conscientiousness than for people of high conscientiousness. Further, we also found significant moderating effects of agreeableness on the relationships of interpersonal conflict and organizational constraints with $\mathrm{CWB}-\mathrm{O}$ and CWB-P, which was predicted by Bowling and Eschleman (2010) but they only found significant results for the organizational constraints-CWB-O relationship and failed on the other relationships. Our results provided further evidence on the moderating role of individual personality traits on relationships of job stressors with CWB, suggesting that people of different levels of individual personality traits (agreeableness and conscientiousness in this case) tend to react to experienced stressors with different levels of CWB.

We found several surprising two-way interactions in the hierarchical regression analyses involving emotional stability. For example, the positive relationships of stressors with CWB were stronger for people of high emotional stability instead of low emotional stability, which was inconsistent with findings of Bowling and Eschleman (2010). Further, the negative relationships of emotional stability with CWB were stronger for people of high conscientiousness instead of low conscientiousness, which was inconsistent with the findings of Jensen and Patel (2011). To better understand these effects, we conducted additional moderation analyses for these moderations by including only one two-way interaction in each of the analyses, but failed to find any significant moderating effect, thus failing to replicate the results reported by Bowling et al. (2011) and Jensen and Patel (2011) involving emotional stability. One possible explanation is that the moderating effect of emotional stability on stressor-CWB relationships are not as straightforward as expected, and its effect might depend on other factors, such as restricting effects of agreeableness and conscientiousness. That is, the moderating effect of emotional stability might only appear when combined with certain levels of other personality traits, which will be further explained with results from threeway interactions.

Based on previous literature (e.g., Bowling et al., 2011; Bowling \& Eschleman, 2010; Grant \& Langan-Fox, 2006; Jensen \& Patel, 2011; Vollrath \& Torgerse, 2000), we proposed that the positive relationships of interpersonal conflict and organizational constraints with CWB will be strongest for people of low emotional stability-low agreeableness among people of all emotional stability-agreeableness combinations, and the hypothesis was supported for interpersonal conflict but not for organizational constraints. Bowling et al. (2011) suggested that although people of low emotional stability are more likely to engage in CWB, high agreeableness will weaken this tendency while low agreeableness will strengthen it. Our results indicated that the same restricting effect of high agreeableness on low emotional stability also works on the interpersonal conflict-CWB relationship, but not on the organizational constraints-CWB relationship. That is, the amplifying effect of low emotional stability on the interpersonal conflict-CWB relationship was biggest when combined with low agreeableness. One possible explanation for the restricting effect is that agreeableness is more concerned with interpersonal relationships and will influence how people handle interpersonal stressors. When people of low emotional stability experience interpersonal job stressors (e.g., interpersonal conflict), they will engage in CWB as a way of coping (Bowling and Eschleman, 2010). However, high agreeableness will restrict the effect of low emotional stability by encouraging people to pay more attention to sustaining good interpersonal relationships and thus motivating them to take more effective coping strategies to deal with the issue, and reduce the tendency to engage in CWB. On the other hand, low agreeableness does not have the restricting effect and thus people of both low emotional stability and low agreeableness will engage in the most CWB. The same regulating effect of agreeableness did not hold for organizational constraints, which might be due to the fact that organizational constraints mainly do not occur interpersonally. When people of low emotional stability experience organizational constraints, their tendency of 
engaging in CWB to cope with the stressor will not be as influenced by their motivation to sustain good interpersonal relationships reflected in high agreeableness, and thus the three-way interaction will not hold up.

Our second hypothesis proposed that the positive relationships of interpersonal conflict and organizational constraints with CWB will be stronger for people of low emotional stability-low conscientiousness than people of other emotional stabilityconscientiousness combinations. Unexpectedly, our results only showed a significant three-way interaction for organizational constraints, and it was in the opposite direction to what we hypothesized. Specifically, our results showed that the positive relationships of organizational constraints with CWB-O and CWB-P were strongest for people of high emotional stability and low conscientiousness among all emotional stability-conscientiousness combinations. Bowling et al. (2011) reported that people of low emotional stability and low conscientiousness engaged in more CWB in general, but it seems that this tendency was not demonstrated in the current study when they face job stressors. It shows that, based on our simple slope analysis results and patterns in Figures 3 and 4, organizational constraints-CWB relationships were weak for people of high conscientiousness regardless of their level of emotional stability, while the relationships were stronger for people of high emotional stability than for people of low emotional stability when combined with low conscientiousness. It is possible that the moderating effect of emotional stability on organizational constraints-CWB relationship can only be expressed when combined with low conscientiousness. When experiencing organizational constraints, low conscientiousness plays an important role in increasing employees' tendency to CWB, and this tendency is strengthened when they are also high on emotional stability. Meanwhile, the nonsignificant interaction of interpersonal conflict, emotional stability, and conscientiousness suggests that effects of combinations of emotional stability and conscientiousness are complicated and need more future studies to address them.

\subsection{Implications}

The current study has several practical implications. When certain job stressors cannot be reduced in the workplace, personality tests involving different personality traits are encouraged to be included in selection processes and the combination of scores on different personality traits can help rule out people who are most likely to engage in CWB after they are recruited. For example, if interpersonal conflict is common in an organization, applicants of low emotional stability and low agreeableness should be selected with caution. Further, organizations should also pay special attention to the interactions of personality and job demands in employee placement decisions. For example, it will benefit the organization to put employees of low agreeableness and low emotional stability in a friendlier environment.

\subsection{Limitations}

The current study has a few limitations that should be considered. First, in the current study all variables are based on self-reports, which might raise concerns for common method variance. While it is possible that common biases might have distorted correlations, this does not seem likely. For example, in a recent metaanalysis, Berry, Carpenter, and Barratt (2012) compared the relationships of self-reported CWB and otherreported CWB with a list of common correlates and found similar patterns between self-reports and otherreports, indicating that use of self-reported CWB did not influence the observed relationships of CWB with other variables. Further, it is likely that self-report of CWB is more accurate because participants might have engaged in some CWBs when they were alone (Bowling et al., 2011), and a different source of report might not be able to capture these behaviors. Because all participants were ensured of the anonymous nature of the survey, it is likely that self-report of CWB provided a more accurate estimate of CWB in which they engaged. Furthermore, it has been suggested that results of moderated multiple regression are unlikely to be influenced by common method variance (Siemsen, Roth, \& Oliveira, 2010), which is the case in the current study. Lastly, emotional stability was included in both hierarchical regression analyses so that we actually controlled emotional stability in the analyses. As emotional stability is closely related to negative affectivity (Milam, Spitzmueller, \& Penney, 2009), controlling emotional stability should help reduce concerns about common method variance. Taken together, using self-report of CWB seems an acceptable practice and this strategy has been taken in recent CWB literature (Bowling et al., 2011; Bowling \& Eschleman, 2010). Nevertheless, studies with multiple sources are encouraged to better address our questions.

Another limitation concerns the cross-sectional design of the current study, and no causal conclusions can be made concerning the relationship we have found. While having a time separation can address this concern, it is possible that the observed relationships will be similar between cross-sectional and longitudinal designs. In a recent study, Meier and Spector (2013) found that there was reciprocal relationship between organizational constraints and CWB, thus collecting stressors and $C W B$ at different times might not change the observed relationships. Nevertheless, we encourage future research to use longitudinal designs to better under- 
stand the underlying mechanism and direction of the observed relationships in the current study.

The third limitation concerns the nature of our sample which was mainly employed students. Although we only included participants who worked at least $20 \mathrm{hr}$ per week, the sample might not be a representative sample of the general working population. To eliminate the effect of part-time employment, we conducted our analyses with and without controlling number of hours in the analyses and the results showed the same pattern. Further, Fox et al. (2001) found that results concerning CWB were not different between samples collected on versus off campus in the community. Furthermore, our zero-order correlations showed relationships of job stressors and personality with CWB that are consistent with previous meta-analyses (Berry et al., 2007; Hershcovis et al., 2007). Taken together, it is unlikely that the nature of our sample could bias the results observed in the current study.

Lastly, we only examined the interactive effects of agreeableness, conscientiousness, and emotional stability on the stressor-CWB relationship while exuding extraversion and openness to experience because they were found to not relate to CWB in past meta-analyses (Berry et al., 2012; Salgado, 2002). However, it is possible that extraverted individuals are more likely to obtain social support, and that high extraversion might inhibit the negative effect of low emotional stability on the stressor-CWB relationship. ${ }^{1}$ Therefore, we encourage future research to explore the interactive effects of additional personality traits on the stressor-CWB relationship.

\subsection{Conclusions}

Past research has well established main effects of job stressors and personality traits, interactive effects between job stressors and personality traits, as well as interactive effects among personality traits in predicting CWB. Our main contribution is incorporating existing approaches to show that the interaction among personality traits also has effect on the stressor-CWB relationships. Our findings show that positive relationship between interpersonal conflict and CWB is strongest for people of low emotional stability-low agreeableness among all emotional stability-agreeableness combinations, while the positive relationship between organizational constraints and CWB is strongest for people of high emotional stability-low conscientiousness among all emotional stability-conscientiousness combinations. Our results support the idea that stressor-CWB relationships are not only influenced by individual personality traits, but also interactions among them. Future research is needed to further understand the underlying mechanisms.

\section{Acknowledgement}

Laurenz L. Meier was supported by Swiss National Science Foundation Grant PAOOP1-131482 and PZ00P1142393.

\section{Note}

1. We would like to thank an anonymous reviewer for pointing this out.

\section{References}

Aiken, L. S., \& West, S. G. (1991). Multiple regression: Testing and interpreting interactions. Newbury Park: Sage.

Berry, C. M., Carpenter, N. C., \& Barratt, C. L. (2012). Do other-reports of counterproductive work behavior provide an incremental contribution over self-reports? A metaanalytic comparison. Journal of Applied Psychology, 97, 613636.

Berry, C. M., Ones, D. S., \& Sackett, P. R. (2007). Interpersonal deviance, organizational deviance, and their common correlates: A review and meta-analysis. Journal of Applied Psychology, 92, 410-424.

Bowling, N. A., Burns, G. N., Stewart, S. M., \& Gruys, M. L. (2011). Conscientiousness and agreeableness as moderators of the relationship between neuroticism and counterproductive work behaviors: A constructive replication. International Journal of Selection and Assessment, 19, 320-330.

Bowling, N. A., \& Eschleman, K. J. (2010). Employee personality as a moderator of the relationships between work stressors and counterproductive work behavior. Journal of Occupational Health Psychology, 15, 91-103.

Camara, W. J., \& Schneider, D. L. (1994). Integrity tests: Facts and unresolved issues. American Psychologist, 49, 112-119.

Cullen, M. J., \& Sackett, P. R. (2003). Personality and counterproductive workplace behavior. In M. Barrick \& A. M. Ryan (Eds.), Personality and work (pp. 150-182). New York: JosseyBass Pfeiffer.

Dawson, J. F., \& Richter, A. W. (2006). Probing three-way interactions in moderated multiple regression: Development and application of a slope difference test. Journal of Applied Psychology, 91, 917-926.

Epstein, S. (1994). Trait theory as personality theory: Can a part be as great as the whole? Psychological Inquiry, 5, 120122.

Fox, S., Spector, P. E., \& Miles, D. (2001). Counterproductive work behavior (CWB) in response to job stressors and organizational justice: Some mediator and moderator tests for autonomy and emotions. Journal of Vocational Behavior, 59, 291-309.

Goldberg, L. R., Johnson, J. A., Eber, H. W., Hogan, R., Ashton, M. C., Cloninger, C. R. et al. (2006). The International Personality Item Pool and the future of public-domain personality measures. Journal of Research in Personality, 40, 8496.

Grant, S., \& Langan-Fox, J. (2006). Occupational stress, coping and strain: The combined/interactive effect of the Big Five traits. Personality and Individual Differences, 41, 719-732. 
Hershcovis, M. S., Turner, N., Barling, J., Arnold, K. A., Dupre, K. E., Inness, M. et al. (2007). Predicting workplace aggression: A meta-analysis. Journal of Applied Psychology, 92, 228238.

Jensen, J. M., \& Patel, P. C. (2011). Predicting counterproductive work behavior from the interaction of personality traits. Personality and Individual Differences, 51, 466-471.

Meier, L. L., \& Spector, P. E. (2013). Reciprocal effects of work stressors and counterproductive work behavior: A fivewave longitudinal study. Journal of Applied Psychology, 98, 529-539.

Milam, A. C., Spitzmueller, C., \& Penney, L. M. (2009). Investigating individual differences among targets of workplace incivility. Journal of Occupational Health Psychology, 14, 58-69.

Penney, L. M. (2002). Workplace incivility and counterproductive work behavior (CWB): What is the relationship and does personality play a role? Doctoral Dissertation, University of South Florida, Tampa.

Penney, L. M., \& Spector, P. E. (2005). Job stress, incivility, and counterproductive work behavior (CWB): The moderating role of negative affectivity. Journal of Organizational Behavior, 26, 777-796.

Robinson, S. L., \& Bennett, R. J. (1995). A typology of deviant workplace behaviors: A multidimensional scaling study. Academy of Management Journal, 38, 555-572.

Rotundo, M., \& Spector, P. E. (2010). Counterproductive work behavior and withdrawal. In J. L. Farr \& N. T. Tippins (Eds.), Handbook of employee selection (Pp. 489-511). New York, NY: Routledge/Taylor \& Francis Group.

Sackett, P. R., \& DeVore, C. J. (2002). Counterproductive behaviors at work. In N. Anderson, D. S. Ones, H. K. Sinangil
\& C. Viswesvaran (Eds.), Handbook of industrial, work and organizational psychology, volume 2001: Personnel psychology (pp. 2145-2164). Thousand Oaks, CA: Sage Publications Ltd.

Salgado, J. F. (2002). The Big Five personality dimensions and counterproductive behaviors. International Journal of Selection and Assessment, 10, 117-125.

Siemsen, E., Roth, A., \& Oliveira, P. (2010). Common method bias in regression models with linar, quadractic, and interaction effects. Organizational Research Methods, 13, 456476.

Spector, P. E., \& Fox, S. (2005). The stressor-emotion model of counterproductive work behavior. In P. E. Spector \& S. Fox (Eds.), Counterproductive work behavior: Investigations of actors and targets (pp. 151-174). Washington, DC: American Psychological Association.

Spector, P. E., Fox, S., Penney, L. M., Bruursema, K., Goh, A., \& Kessler, S. (2006). The dimensionality of counterproductivity: Are all counterproductive behaviors created equal? Journal of Vocational Behavior, 68, 446-460.

Spector, P. E., \& Jex, S. M. (1998). Development of four selfreport measures of job stressors and strain: Interpersonal Conflict at Work Scale, Organizational Constraints Scale, Quantitative Workload Inventory, and Physical Symptoms Inventory. Journal of Occupational Health Psychology, 3, 356367.

Vardi, Y., \& Weitz, E. (2004). Misbehavior in organizations. Mahwah, NJ: Erlbaum.

Vollrath, M., \& Torgerse, S. (2000). Personality types and coping. Personality and Individual Differences, 29, 367378. 